\title{
Effect of double stratification on mixed convection boundary layer flow of a nanofluid past a vertical plate in a porous medium
}

\author{
D. Srinivasacharya $\cdot$ Ontela Surender
}

Received: 3 November 2013/Accepted: 30 December 2013/Published online: 12 January 2014

(C) The Author(s) 2014. This article is published with open access at Springerlink.com

\begin{abstract}
The effect of thermal and mass stratification on mixed convection boundary layer flow over a vertical flat plate embedded in a porous medium saturated by a nanofluid has been investigated. The vertical plate is maintained at uniform and constant heat, mass and nanoparticle fluxes, and the behavior of the porous medium is described by the Darcy model. The model considered for nanofluids incorporates the effects of Brownian motion and thermophoresis. In addition, the thermal energy equations include regular diffusion and cross-diffusion terms. A suitable coordinate transformation is introduced, and the obtained system of non-similar, coupled and non-linear partial differential equations is solved numerically. The influence of pertinent parameters on the non-dimensional velocity, temperature, concentration and nanoparticle volume fraction are discussed. In addition, the variation of heat, mass and nanoparticle transfer rates at the plate are exhibited graphically for different values of physical parameters.
\end{abstract}

Keywords Mixed convection - Nanofluid - Thermal stratification - Solutal stratification · Brownian motion . Thermophoresis

\section{Introduction}

During the past decade, the study of nanofluids has attracted enormous interest from research due to its exceptional applications to electronics, communication, computing technologies, optical devices, lasers, high-

D. Srinivasacharya $(\bowtie) \cdot$ O. Surender

Department of Mathematics, National Institute of Technology,

Warangal 506004, Andhra Pradesh, India

e-mail: dsrinivasacharya@yahoo.com; dsc@nitw.ac.in power X-rays, scientific measurement, material processing, medicine and material synthesis. Nanofluids are prepared by dispersing solid nanoparticles in base fluids such as water, oil or ethylene glycol. Choi et al. (2011) showed that the addition of a small amount $(<1$ by volume) of nanoparticles to conventional heat transfer liquids increased the thermal conductivity of the fluid. The detailed introduction and applications of nanofluids can be found in the book by Das et al. (2007). Buongiorno (2006) has investigated the factors that contribute to abnormal thermal conductivity increase relative to base fluids and viscosity. He developed an analytical model for convective transport in nanofluids, which takes Brownian diffusion and thermophoresis effects into account. The random motion of nanoparticles within the base fluid is called Brownian motion, and this results from continuous collisions between the nanoparticles and the molecules of the base fluid. Particles can diffuse under the effect of a temperature gradient. This phenomenon is called thermophoresis, and is the particle equivalent of the well-known Soret effect for gaseous or liquid mixtures. The literature on nanofluids has been reviewed by Kakac and Pramuanjaroenkij (2009), Gianluca et al. (2011) among several others. These reviews discuss in detail the work done on convective transport in nanofluids.

Convective transport in porous media has been the subject of great importance and interest in the recent years owing to its wide range of applications in civil, chemical and mechanical engineering. Several authors investigated the mixed convection heat and mass transfer along nonisothermal vertical surface embedded in a nanofluid saturated porous medium. Nazar et al. (2011) considered the steady mixed convection boundary layer flow from an isothermal horizontal circular cylinder embedded in a porous medium filled with a nanofluid for both cases of a heated and cooled cylinder. Rosca et al. (2012) studied 
numerically the problem of steady mixed convection boundary layer flow over an impermeable horizontal flat plate embedded in a porous medium saturated by a nanofluid. Rana et al. (2012) analyzed the steady mixed convection boundary layer flow of an incompressible nanofluid along a plate inclined at an angle $a$ in a porous medium. RamReddy et al. (2013) presented the influence of the Soret effect on mixed convection heat and mass transfer in the boundary layer region of a semi-infinite vertical flat plate in a nanofluid under the convective boundary conditions. Rohni et al. (2013) studied the steady mixed convection boundary layer flow on a vertical circular cylinder embedded in a porous medium filled by a nanofluid. Chamkha et al. (2013) studied the problem of steady, laminar, mixed convection boundary layer flow over a vertical cone embedded in a porous medium saturated with a nanofluid in the presence of thermal radiation taking into account the effects of Brownian motion and thermophoresis with Rosseland diffusion approximation. Heat transfer characteristics of a two-dimensional steady hydromagnetic natural convection flow of nanofluids over a non-linear stretching sheet taking into account the effects of radiation and convective boundary condition have been investigated numerically by Rahman and Eltayeb (2013).

In all the above mentioned studies, the effect of stratification has been neglected. Stratification is a formation/deposition of layers which occur due to temperature variations, concentration differences, or the presence of different fluids. In practical situations where the heat and mass transfer mechanisms run simultaneously, it is interesting and important to analyze the influence of double stratification (stratification of medium with respect to thermal and concentration fields) on the convective transport in nanofluid. The analysis of natural and mixed convection in a doubly stratified medium is a fundamentally interesting and important problem, because of its broad range of engineering applications. These applications include heat rejection into the environment such as lakes, rivers, and seas; thermal energy storage systems such as solar ponds; and heat transfer from thermal sources such as the condensers of power plants. Rosmila et al. (2012) examined the magnetohydrodynamic convection flow and heat transfer of an incompressible viscous nanofluid past a semi-infinite vertical stretching sheet in the presence of thermal stratification. They have concluded that the flow field, temperature, and nanoparticle volume fraction profiles are significantly influenced by the thermal stratification and the magnetic field.

Previous studies on convection transport focussed on seeking similarity solution, because similar variables can give great physical insight with minimal effort. However, the non-similarity boundary layer flows are more general in nature in our everyday life, and thus are more important than the similarity ones. It may be remarked that earlier studies did not include the effect of double stratification on double diffusive mixed convection flow of a nanofluid past a vertical plate in a porous medium. Hence, in the present study, we have made an attempt to study the effect of double stratification on mixed convection heat and mass transfer over a vertical plate in a nanofluid-saturated porous medium. The governing system of non-linear partial differential equations are solved numerically using implicit finite difference method. The influence of pertinent parameters on the flow characteristics is examined and exhibited through graphs.

\section{Mathematical formulation}

Consider the mixed convection boundary layer flow over a semi-infinite vertical flat plate embedded in a porous medium saturated with a nanofluid. The $x$ coordinate is taken along the plate, in the ascending direction and the $y$ coordinate is measured normal to the plate, while the origin of the reference system is considered at the leading edge of the vertical plate. The plate has the constant wall heat, mass and nanoparticle fluxes. The ambient medium is assumed to be vertically linearly stratified with respect to both temperature and concentration in the form $T_{\infty}(x)=T_{\infty, 0}+A x, C_{\infty}(x)=C_{\infty, 0}+B x$, where $A$ and $B$ are constants which are varied to alter the intensity of stratification in the medium, $T_{\infty, 0}$ and $C_{\infty, 0}$ are ambient temperature and concentration, respectively. The values of $T_{\mathrm{w}}$ and $C_{\mathrm{w}}$ are assumed to be greater than the ambient temperature $T_{\infty, 0}$ and concentration $C_{\infty, 0}$ at any arbitrary reference point in the medium (inside the boundary layer). The nanoparticle volume fraction on the surface of the plate is $\phi_{\mathrm{w}}$, and the ambient value of the nanoparticle volume fraction is denoted by $\phi_{\infty}$. The porous medium is assumed to be uniform and isotropic and is in local thermal equilibrium with the fluid. The effects of Brownian motion and thermophoresis are incorporated into the model for nanofluids. The fluid properties are assumed to be constant except for density variations in the buoyancy force term.

Using the Boussinesq and boundary layer approximations, the governing equations for the conservation of total mass, momentum, energy and nanoparticles within the boundary layer near the vertical plate can be written as:

$\frac{\partial u}{\partial x}+\frac{\partial v}{\partial y}=0$ 


$$
\begin{aligned}
& \rho_{\mathrm{f}}\left(u \frac{\partial u}{\partial x}+v \frac{\partial u}{\partial y}\right)=\mu \frac{\partial^{2} u}{\partial y^{2}}+\left(1-\phi_{\infty}\right) \rho_{\mathrm{f}_{\infty}} g^{*}\left(\beta_{\mathrm{T}}\left(T-T_{\infty}\right)\right. \\
& \left.+\beta_{\mathrm{C}}\left(C-C_{\infty}\right)\right)-\left(\rho_{\mathrm{p}}-\rho_{\mathrm{f}_{\infty}}\right) g^{*}\left(\phi-\phi_{\infty}\right)-\frac{\mu}{K_{\mathrm{p}}}\left(u-u_{\infty}\right) \\
& u \frac{\partial T}{\partial x}+v \frac{\partial T}{\partial y}=\alpha \frac{\partial^{2} T}{\partial y^{2}}+\tau\left[D_{\mathrm{B}} \frac{\partial \phi}{\partial y} \frac{\partial T}{\partial y}+\frac{D_{\mathrm{T}}}{T_{\infty}}\left(\frac{\partial T}{\partial y}\right)^{2}\right] \\
& +D_{\mathrm{TC}} \frac{\partial^{2} C}{\partial y^{2}} \\
& u \frac{\partial C}{\partial x}+v \frac{\partial C}{\partial y}=D_{\mathrm{s}} \frac{\partial^{2} C}{\partial y^{2}}+D_{\mathrm{CT}} \frac{\partial^{2} T}{\partial y^{2}} \\
& u \frac{\partial \phi}{\partial x}+v \frac{\partial \phi}{\partial y}=D_{\mathrm{B}} \frac{\partial^{2} \phi}{\partial y^{2}}+\frac{D_{\mathrm{T}}}{T_{\infty}} \frac{\partial^{2} T}{\partial y^{2}}
\end{aligned}
$$

where $u$ and $v$ are the components of velocity along $x$ and $y$ directions, respectively, $g^{*}$ is the gravitational acceleration, $T$ is the temperature, $C$ is the concentration, $\phi$ is the nanoparticle volume fraction, $\rho_{\mathrm{f}}$ is density of the base fluid, $\rho_{\mathrm{p}}$ is the density of nanoparticles, $K_{\mathrm{p}}$ is the Permeability, $\beta_{\mathrm{T}}$ and $\beta_{\mathrm{c}}$ are volumetric thermal and solutal expansion coefficients of nanofluid, respectively. $\mu$ is the dynamic coefficient of viscosity of the fluid, $v$ is the kinematic viscosity, $\alpha$ is the thermal diffusivity, $D_{\mathrm{s}}$ is the solutal diffusivity, $D_{\mathrm{B}}$ is the Brownian diffusion coefficient, $D_{\mathrm{T}}$ is the Thermophoretic diffusion coefficient. $D_{\mathrm{TC}}$ and $D_{\mathrm{CT}}$ are Dufour and Soret type diffusivities, respectively. The boundary conditions are:

$$
\left.\begin{array}{c}
u=0, v=0, q_{\mathrm{w}}=-k\left(\frac{\partial T}{\partial y}\right)_{y=0}, q_{\mathrm{m}}=-D_{s}\left(\frac{\partial C}{\partial y}\right)_{y=0}, \\
q_{\mathrm{np}}=-D_{\mathrm{B}}\left(\frac{\partial \phi}{\partial y}\right)_{y=0} \text { at } \quad y=0
\end{array}\right\}
$$

$$
\underset{y \rightarrow u_{\infty}, T \rightarrow T_{\infty}(x), C \rightarrow C_{\infty}(x), \phi \rightarrow \phi_{\infty}(x) \text { as }}{u \rightarrow \infty}
$$

where the subscripts $w$ and $\infty$ indicates the conditions at wall and at the outer edge of the boundary layer, respectively.

The continuity equation $(\sim 1)$ is satisfied by introducing the stream function $\psi$ such that

$u=\frac{\partial \psi}{\partial y}, \quad v=-\frac{\partial \psi}{\partial x}$

Introducing the following non-dimensional variables

$$
\left.\begin{array}{c}
\xi=\frac{x}{L}, \eta=\frac{R e^{1 / 2}}{L \xi^{1 / 2}} y, \psi=R e^{-1 / 2} \xi^{1 / 2} L u_{\infty} f(\xi, \eta) \\
T-T_{\infty}(x)=\frac{q_{\mathrm{w}} L}{k \operatorname{Re}^{1 / 2}} \xi^{1 / 2} \theta(\xi, \eta) \\
C-C_{\infty}(x)=\frac{q_{\mathrm{m}} L}{D_{\mathrm{s}} \operatorname{Re} e^{1 / 2}} \xi^{1 / 2} s(\xi, \eta) \\
\phi-\phi_{\infty}(x)=\frac{q_{\mathrm{np}} L}{D_{\mathrm{B}} \operatorname{Re} e^{1 / 2}} \xi^{1 / 2} g(\xi, \eta)
\end{array}\right\}
$$

into Eqs. (2-5), we obtain

$$
\begin{aligned}
& f^{\prime \prime \prime}+\frac{1}{2} f f^{\prime \prime}-\frac{\xi}{D a R e}\left(f^{\prime}-1\right)+R i \xi^{3 / 2}\left(\theta+B_{\mathrm{s}}-N_{\mathrm{r}} g\right) \\
& =\xi\left[f^{\prime} \frac{\partial f^{\prime}}{\partial \xi}-f^{\prime \prime} \frac{\partial f}{\partial \xi}\right] \\
& \frac{1}{P r}\left(\theta^{\prime \prime}+N_{\mathrm{b}} \xi^{1 / 2} g^{\prime} \theta^{\prime}+N_{\mathrm{t}} \xi^{1 / 2}\left(\theta^{\prime}\right)^{2}+N_{\mathrm{d}} s^{\prime \prime}\right)+\frac{1}{2} f \theta^{\prime} \\
& \quad-\frac{1}{2} f^{\prime} \theta-\epsilon_{1} \xi^{1 / 2} f^{\prime}=\xi\left[f^{\prime} \frac{\partial \theta}{\partial \xi}-\theta^{\prime} \frac{\partial f}{\partial \xi}\right] \\
& \frac{1}{S c}\left(s^{\prime \prime}+L_{\mathrm{d}} \theta^{\prime \prime}\right)+\frac{1}{2} f s^{\prime}-\frac{1}{2} f^{\prime} s-\epsilon_{2} \xi^{1 / 2} f^{\prime}=\xi\left[f^{\prime} \frac{\partial s}{\partial \xi}-s^{\prime} \frac{\partial f}{\partial \xi}\right] \\
& \frac{1}{S c_{n}}\left(g^{\prime \prime}+\frac{N_{\mathrm{t}}}{N_{\mathrm{b}}} \theta^{\prime \prime}\right)+\frac{1}{2} f g^{\prime}-\frac{1}{2} f^{\prime} g=\xi\left[f^{\prime} \frac{\partial g}{\partial \xi}-g^{\prime} \frac{\partial f}{\partial \xi}\right]
\end{aligned}
$$

where the prime denotes differentiation with respect to the similarity variable $\eta, B=\frac{\beta_{\mathrm{C}} q_{\mathrm{m}} k}{\beta_{\mathrm{T}} q_{\mathrm{w}} D_{\mathrm{s}}}$ is the regular double diffusive buoyancy ratio, $N_{\mathrm{r}}=\frac{\left(\rho_{\mathrm{p}}-\rho_{\mathrm{f}_{\infty}}\right) q_{\mathrm{np}} k}{\rho_{\mathrm{f}_{\infty}} \beta_{\mathrm{T}}\left(1-\phi_{\infty}\right) q_{\mathrm{w}} D_{\mathrm{B}}}$ is nanofluid buoyancy ratio, $K_{\mathrm{p}}$ is Permeability, $D a=\frac{K_{\mathrm{p}}}{L^{2}}$ is Darcian number, $\operatorname{Pr}=\frac{v}{\alpha}$ is the Prandtl number, $\epsilon_{1}=\frac{k}{q_{\mathrm{w}}} R e^{1 / 2} A$ and $\epsilon_{2}=\frac{D_{\mathrm{s}}}{q_{\mathrm{m}}} R e^{1 / 2} B$ are the thermal and solutal stratification parameters, respectively, $G r=\frac{\left(1-\phi_{\infty}\right) \rho_{\mathrm{f}_{\infty}} g^{*} \beta_{\mathrm{T}} q_{\mathrm{w}} L^{4}}{\mu \nu k}$ is Grashof number, $S c=\frac{v}{D_{\mathrm{s}}}$ is the Schmidth number, $N_{\mathrm{t}}=\frac{\tau D_{\mathrm{T}} q_{\mathrm{w}} L}{\alpha T_{\infty} k R e^{1 / 2}} \quad$ is thermophoresis parameter, $N_{\mathrm{b}}=\frac{\tau q_{\mathrm{np}} L}{\alpha \operatorname{Re}^{1 / 2}}$ is Brownian motion parameter, $S c_{n}=\frac{v}{D_{\mathrm{B}}}$ is nanofluid Schmidt number, $L_{\mathrm{d}}=\frac{D_{\mathrm{CT}} q_{\mathrm{w}}}{q_{\mathrm{m}} k}$ is Dufour-solutal Lewis number and $N_{\mathrm{d}}=\frac{D_{\mathrm{TC}} q_{\mathrm{m}} k}{\alpha D_{\mathrm{s}} q_{\mathrm{w}}} \quad$ is modified Dufour number. The 
corresponding boundary conditions in dimensionless form are:

$$
\begin{gathered}
f^{\prime}(\xi, 0)=0, f(\xi, 0)+2 \xi\left(\frac{\partial f}{\partial \xi}\right)_{\substack{\eta=0\\
}}=0, \theta^{\prime}(\xi, 0) \\
=-1, s^{\prime}(\xi, 0)=-1, g^{\prime}(\xi, 0)=-1 \\
f^{\prime}(\xi, \infty)=1, \quad \theta(\xi, \infty)=0, \quad s(\xi, \infty)=0, \\
g(\xi, \infty)=0
\end{gathered}
$$

The heat, mass and nanoparticle volume fraction transfers from the plate, respectively, are given by

$$
\begin{gathered}
q_{\mathrm{w}}=-k\left(\frac{\partial T}{\partial y}\right)_{y=0} q_{\mathrm{m}}=-D_{\mathrm{s}}\left[\frac{\partial C}{\partial y}\right]_{y=0} \text { and } \\
q_{\mathrm{np}}=-D_{\mathrm{B}}\left[\frac{\partial \phi}{\partial y}\right]_{y=0}
\end{gathered}
$$

The non-dimensional rate of heat transfer, called the Nusselt number $N u_{\xi}=\frac{q_{\mathrm{w}} x}{k\left(T_{\mathrm{w}}-T_{\infty, 0}\right)}$, rate of mass transfer, called the Sherwood number $S h_{\xi}=\frac{q_{\mathrm{m}} x}{D_{\mathrm{s}}\left(C_{\mathrm{w}}-C_{\infty, 0}\right)}$ and nanoparticle volume fraction transfer, called nanofluid Sherwood number $S h_{n, \xi}=\frac{q_{\mathrm{np}} x}{D_{\mathrm{B}}\left(\phi_{\mathrm{w}}-\phi_{\infty}\right)}$ are given by

$$
\begin{aligned}
& \frac{N u_{\xi}}{R e^{1 / 2}}=\frac{\xi^{1 / 2}}{\epsilon_{1} \xi^{1 / 2}+\theta(\xi, 0)}, \quad \frac{S h_{\xi}}{R e^{1 / 2}}=\frac{\xi^{1 / 2}}{\epsilon_{2} \xi^{1 / 2}+s(\xi, 0)} \quad \text { and } \\
& \frac{S h_{n, \xi}}{R e^{1 / 2}}=\frac{\xi^{1 / 2}}{g(\xi, 0)}
\end{aligned}
$$

\section{Results and discussions}

The governing non-linear and non-homogeneous partial differential equations Eqs. (9-12) are solved using the implicit finite-difference scheme discussed in Cebeci and Bradshaw (1984). This method is adequately explained in the literature and it gives accurate results for boundary layer equations. In the present study, the boundary conditions for $\eta$ at $\infty$ are replaced by a sufficiently large value where the velocity, temperature and concentration approach zero. In order to see the effects of step size $(\Delta \eta)$, we ran the code for our model with three different step

Table 1 Convergence analysis with step sizes $\Delta \eta=0.05,0.01,0.001$ for the velocity, temperature, concentration and nanoparticle volume fraction

\begin{tabular}{lllll}
\hline$\Delta \eta$ & $f^{\prime \prime}(\xi, 0)$ & $\theta(\xi, 0)$ & $s(\xi, 0)$ & $g(\xi, 0)$ \\
\hline 0.05 & 0.47843954 & 2.99380339 & 1.97888089 & 2.07132977 \\
0.01 & 0.47843286 & 2.99442992 & 1.97877599 & 2.07146725 \\
0.001 & 0.47843258 & 2.99445577 & 1.97877166 & 2.07147292 \\
\hline
\end{tabular}

sizes as $\Delta \eta=0.001,0.01$ and 0.05 and in each case we found very good agreement between them on different profiles, which is presented in Table. (1). After some trials, we imposed a maximal value of $\eta$ at $\infty$ of 10 and a grid size of $\Delta \eta$ as 0.01 .

In order to assess the accuracy of our method, we have compared our results with those of Lee et al. (1987) in the absence of $\epsilon_{1}, \epsilon_{2}, \xi, N_{\mathrm{r}}, N_{\mathrm{b}}, N_{\mathrm{t}}, L_{\mathrm{d}}$ and $N_{\mathrm{d}}$ with the variation of Prandtl number $P r$. The comparison in the above case is found to be in good agreement, as shown in Table. (2)

The effect of thermal stratification parameter $\varepsilon_{1}$ on nondimensional velocity, temperature, concentration and nanoparticle fractions is shown in Figs. 1, 2, 3, and 4. It is observed from Fig. 1 that the velocity decreases with the increase of thermal stratification $\varepsilon_{1}$. Increase in thermal stratification parameter reduces the effective convective potential between the heated plate and the ambient fluid in the medium. This factor causes a decrease in the buoyancy force, which decelerates the velocity of the flow. It is noticed from Fig. 2 that the non-dimensional temperature of the fluid decreases with the increase of the thermal

Table 2 Comparison between $\frac{1}{\theta(0)}$ calculated by the present method and that of Lee et al. (1987) with the variation of Prandtl number $\mathrm{Pr}$

\begin{tabular}{lll}
\hline $\operatorname{Pr}$ & $\frac{1}{\theta(0)}$ & \\
\cline { 2 - 3 } & Lee et al. (1987) & Present \\
\hline 0.1 & 0.2007 & 0.2009 \\
0.7 & 0.4059 & 0.4059 \\
7.0 & 0.8856 & 0.88564 \\
100.0 & 2.1512 & 2.15222 \\
\hline
\end{tabular}

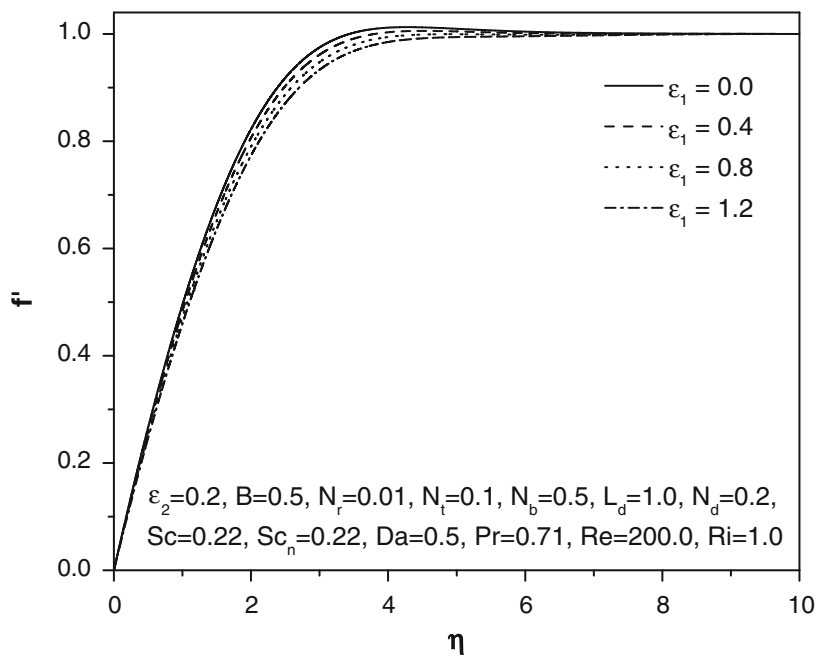

Fig. 1 Velocity profile for various values of thermal stratification parameter 


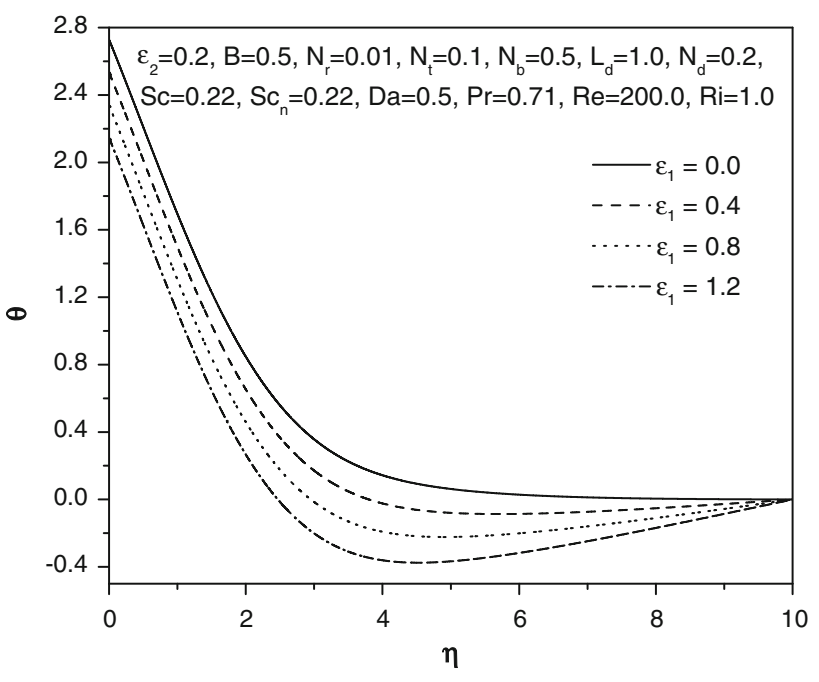

Fig. 2 Temperature profile for various values of thermal stratification parameter

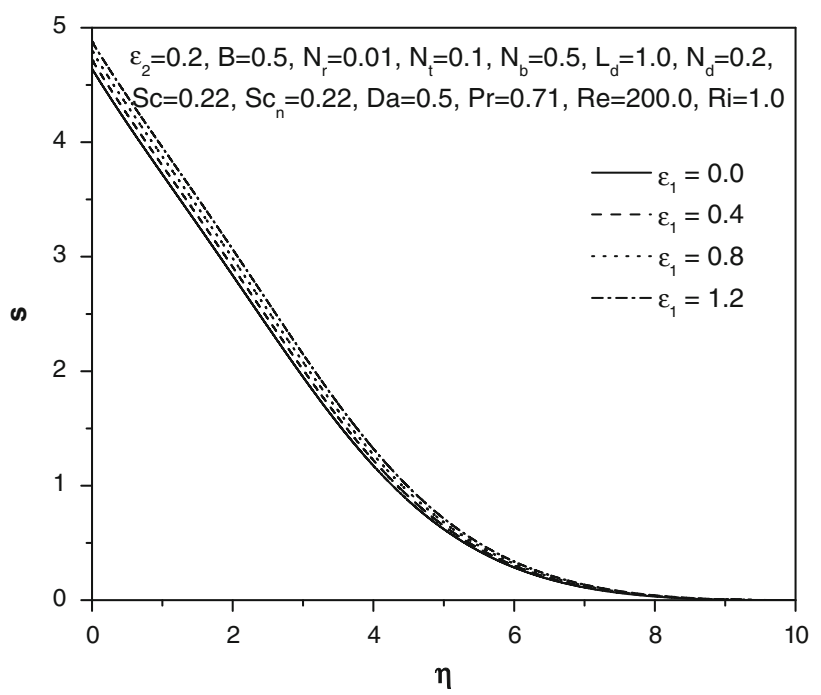

Fig. 3 Concentration profile for various values of thermal stratification parameter

stratification parameter. When the thermal stratification effect is taken into consideration, the temperature gradient between the plate and the ambient fluid will decrease. In view of this, the thermal boundary layer is thickened and the temperature is reduced. The magnitudes of concentration and nanoparticle volume fraction enhance with the increase of thermal stratification parameter as shown in Figs. 3 and 4.

In Figs. 5, 6, 7 and 8 the influence of solutal stratification parameter $\varepsilon_{2}$ on the dimensionless velocity, temperature, concentration and nanoparticle fractions is presented. From Fig. 5, it is observed that the velocity of the fluid decreases with the increase of solutal stratification parameter. Increase in mass stratification parameter lessens the concentration gradient between the

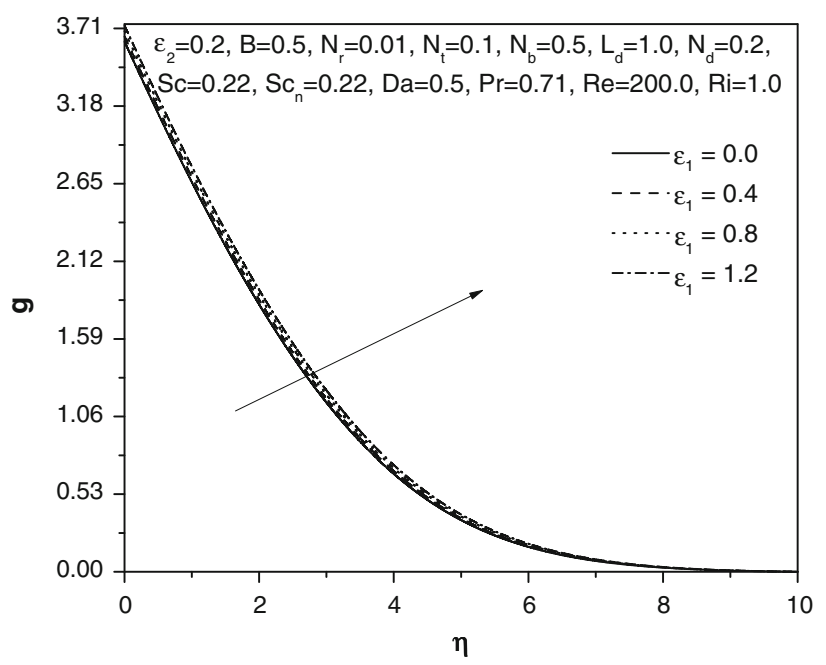

Fig. 4 Nanoparticle volume fraction profile for various values of thermal stratification parameter

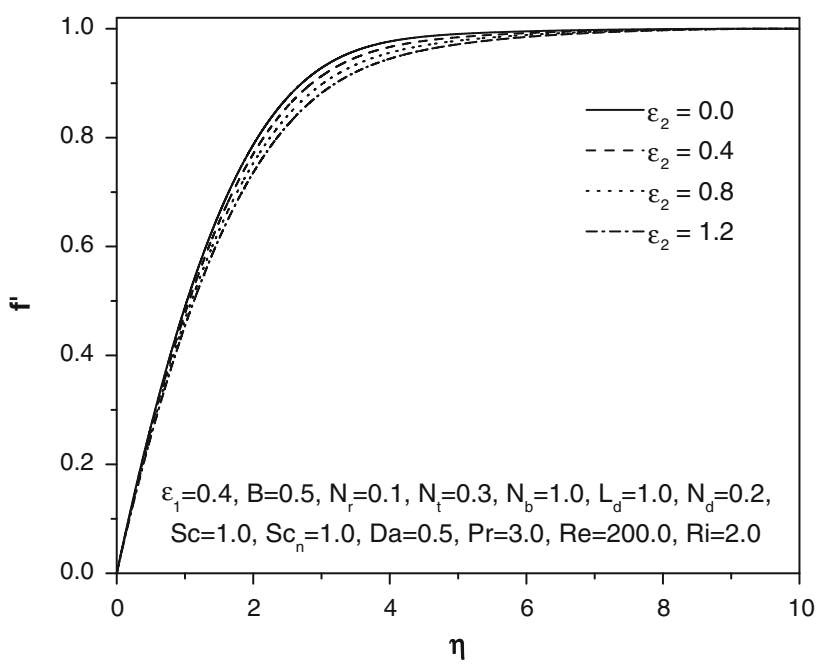

Fig. 5 Velocity profile for various values of solutal stratification parameter

ambient and the surface. This declines the buoyancy force, which reduces the velocity of the flow. It is noticed from Fig. 6 that the temperature of the fluid increases with the increase of solutal stratification parameter. It is clear from Fig. 7 that the non-dimensional concentration of the fluid decreases with the increase of solutal stratification parameter. From Fig. 8, it can be noticed that the solutal stratification parameter enhances the nanoparticle fraction. It is observed that the non-dimensional temperature and concentration values would become negative inside the boundary layer for different values of the stratification parameters depending on the values of other parameters. This is because the fluid near the plate can have temperature or concentration lower than the ambient medium. 


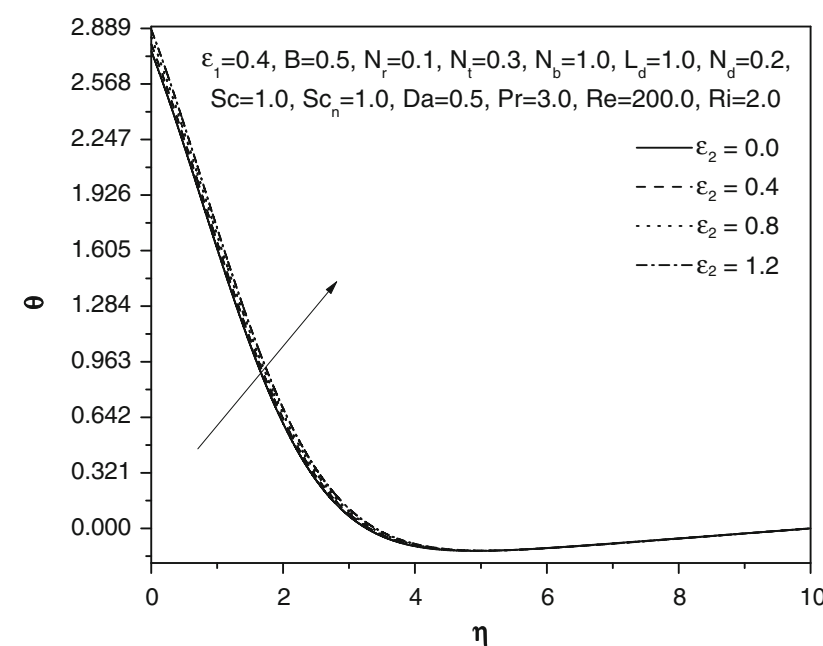

Fig. 6 Temperature profile for various values of solutal stratification parameter

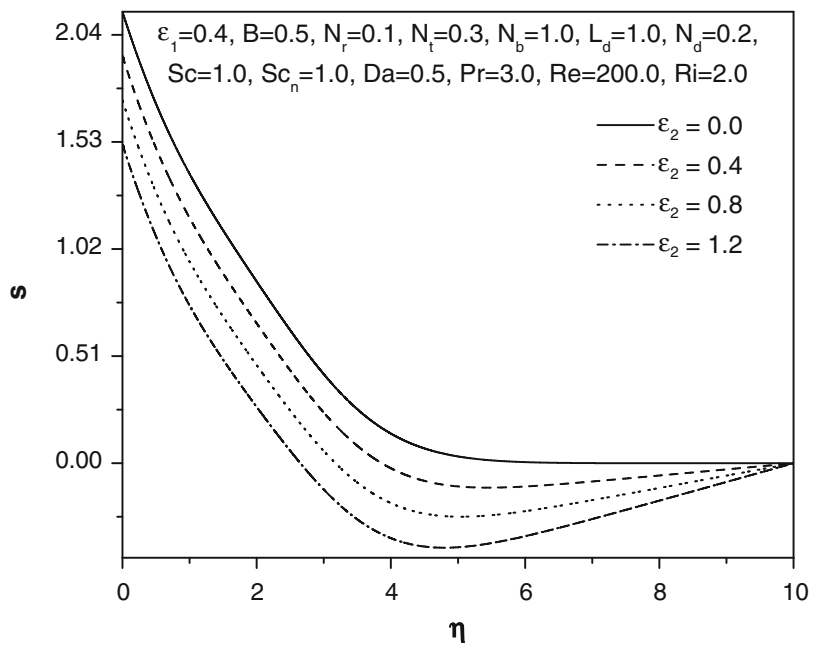

Fig. 7 Concentration profile for various values of solutal stratification parameter

Figures 9, 10 and 11 depict the effect of thermal stratification parameter on the non-dimensional Nusselt number, Sherwood number and nanoparticle Sherwood number. As $\xi$ increases, the Nussellt number, Sherwood number and nanoparticle sherwood number increases. Figures 9, 10 and 11 reveal that an enhancement in thermal stratification reduces heat, mass and nanoparticle transfer rates. Figures 12, 13 and 14 show the effect of solutal stratification parameter on dimensionless Nusselt number, Sherwood number and nanoparticle Sherwood number. As $\xi$ increases, the Nussellt number, Sherwood number and nanoparticle sherwood number increases. An increase in solutal stratification parameter causes a significant decrease in non-dimensional heat, mass and nanoparticle transfer rates as shown in Figs. 12, 13 and 14.

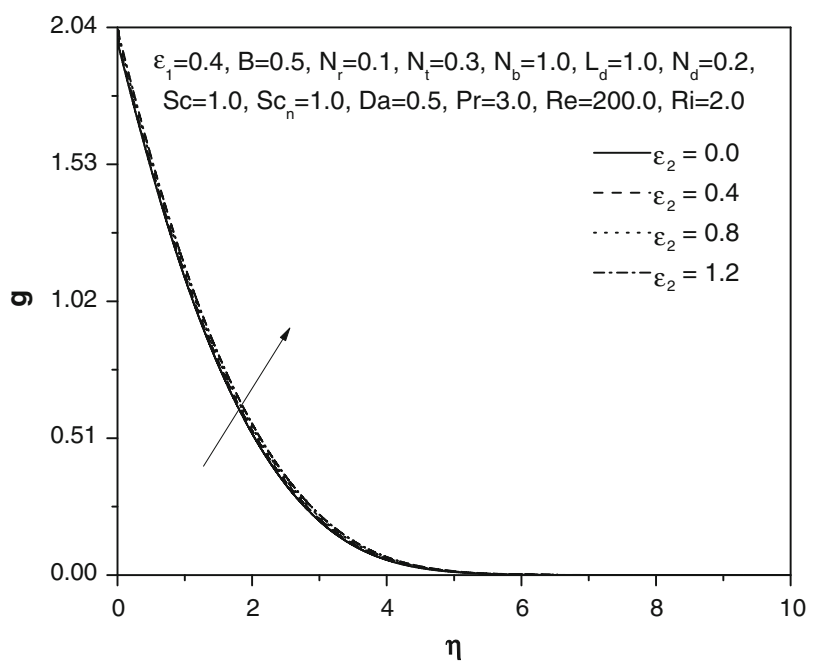

Fig. 8 Nanoparticle volume fraction profile for various values of solutal stratification parameter

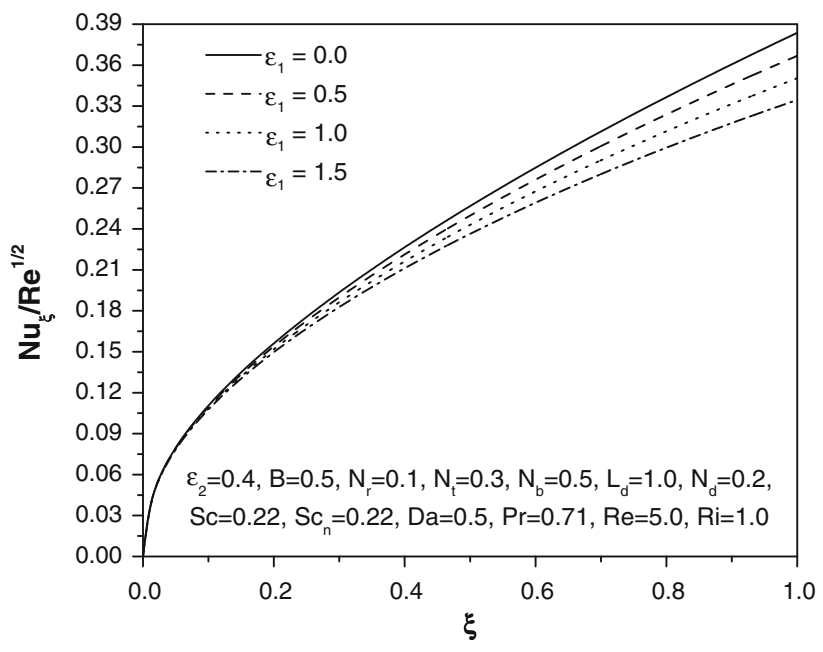

Fig. 9 Variation of local heat transfer coefficient with thermal stratification parameter

The dimensionless heat, mass and nanoparticle volume fraction transfer rates for different values of thermophoresis parameter $N_{\mathrm{t}}$ and Brownian motion parameter $N_{\mathrm{b}}$ are presented in Figs. 15, 16 and 17. Figure 15 depicts that the dimensionless heat transfer rate decreases with the increase in both the thermophoresis and Browinian motion parameters. The dimensionless mass transfer rates increase with the increase of both the thermophoresis and Brownian motion parameters, as shown in Fig. 16. An increase in the value of thermophoresis parameter causes a decrease in nanoparticle volume fraction transfer rate. Further, increasing Brownian motion parameter enhances the nanoparticle volume fraction transfer rate, as shown in Fig. 17.

The variation of dimensionless Nusselt, Sherwood and nanoparticle Sherwood numbers for different values of 


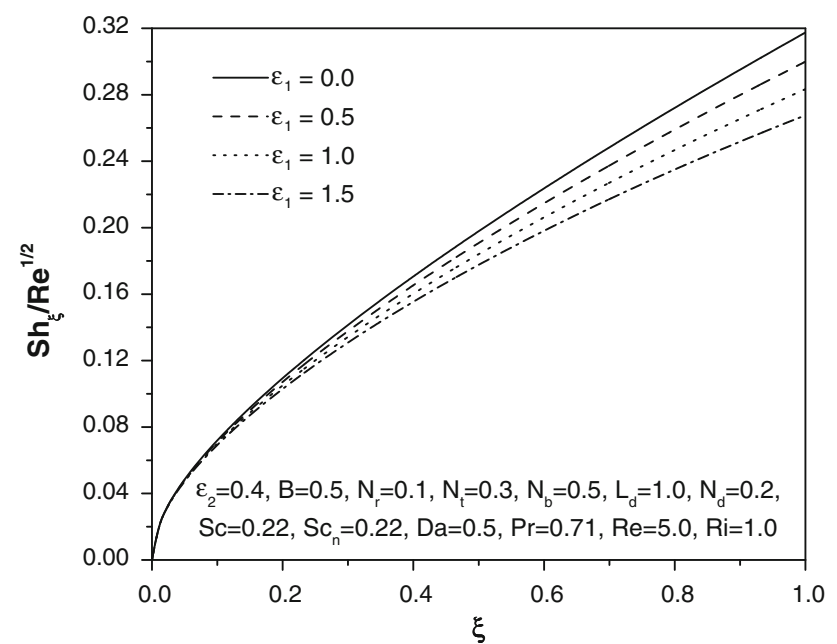

Fig. 10 Variation of local mass transfer coefficient with thermal stratification parameter

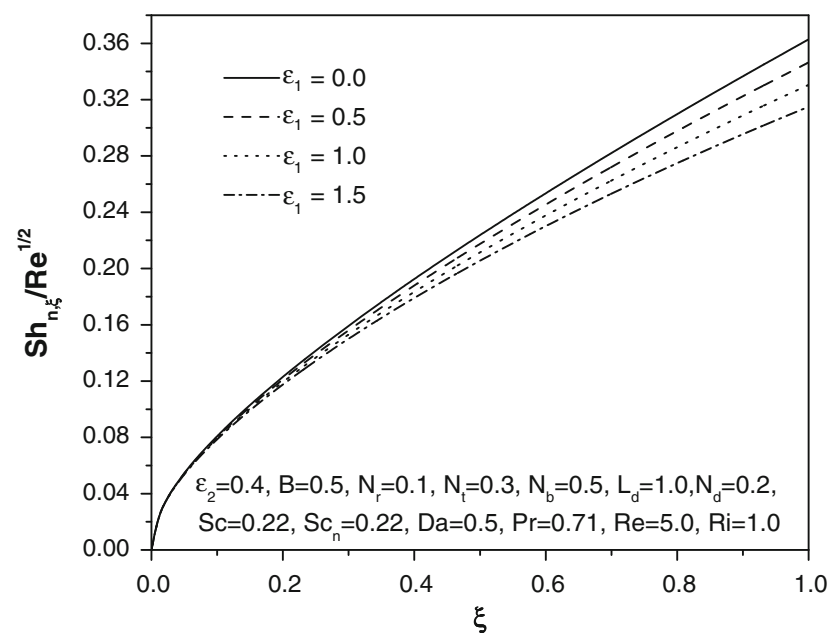

Fig. 11 Variation of local nanoparticle transfer coefficient with thermal stratification parameter

Dufour-solutal Lewis number $L_{\mathrm{d}}$ and modified Dufour number $N_{\mathrm{d}}$ is exhibited in Figs. 18, 19 and 20. It is seen from Fig. 18 that the dimensionless heat transfer rate is increased with the increase in Dufour-solutal Lewis number and decreased with the increase of modified Dufour number. The dimensionless mass transfer rate increases with the increase of modified Dufour number and decreases with an increase in the value of Dufour-solutal Lewis number, as shown in Fig. 19. Figure 20 shows that the nanoparticle volume fraction transfer rate increases with increase in the values of Dufour-solutal Lewis number and modified Dufour number.

The non-dimensional heat, mass and nanoparticle transfer rates for different values of Darcy number are presented in Figs. 21, 22 and 23. An increase in Darcy number decreases dimensionless heat, mass and

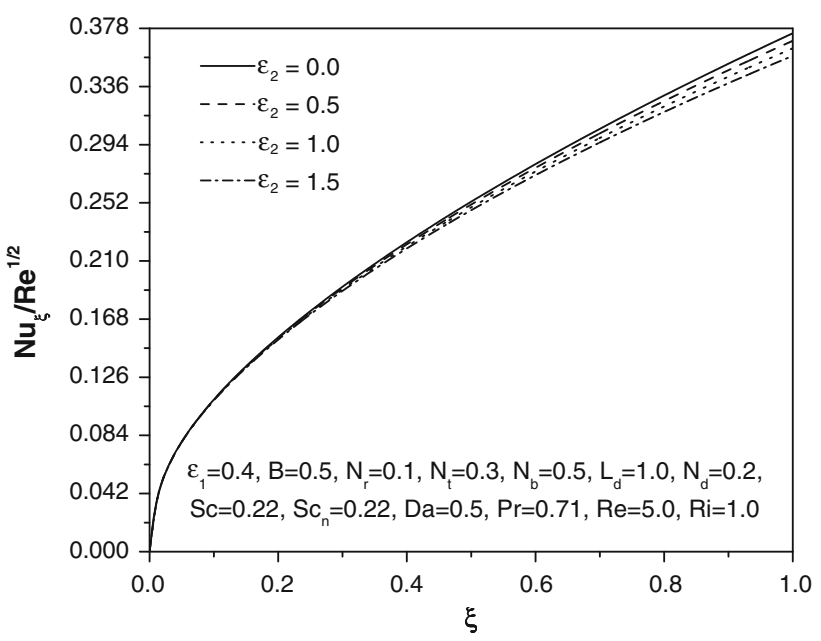

Fig. 12 Variation of local heat transfer coefficient with solutal stratification parameter

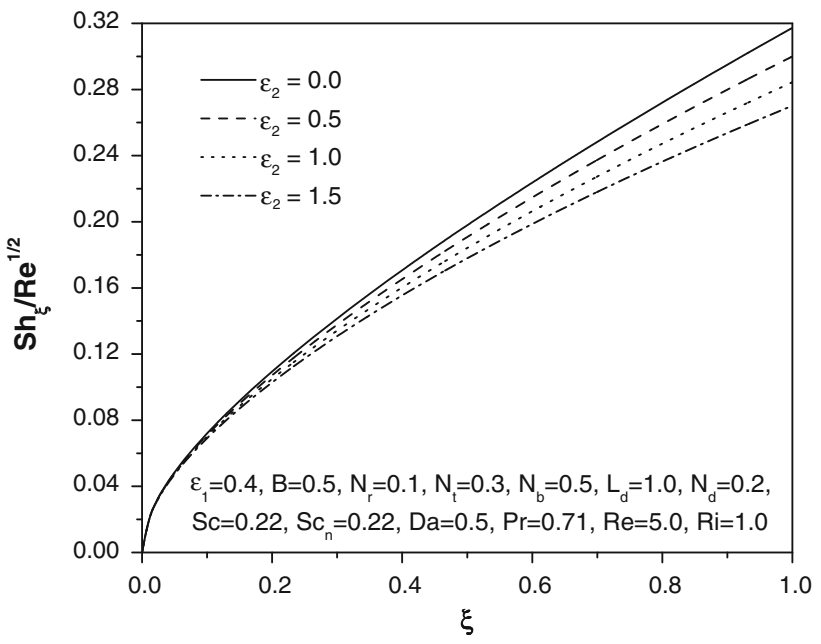

Fig. 13 Variation of local mass transfer coefficient with solutal stratification parameter

nanoparticle transfer rates as depicted in Figs. 21, 22 and 23. Figures 24,25 and 26 present the variation of nondimensional Nusselt, Sherwood and nanoparticle Sherwood numbers with varying mixed convection parameter. The dimensionless heat, mass and nanoparticle transfer rates are enhanced with an increase in the value of mixed convection parameter $R i$ as shown in Figs. 24, 25 and 26.

\section{Conclusions}

In this article, we studied the influence of thermal and solutal stratification on mixed convection boundary layer flow of a nanofluid past a vertical plate embedded in a porous medium. The effects of Brownian motion and thermophoresis are incorporated into the model for 


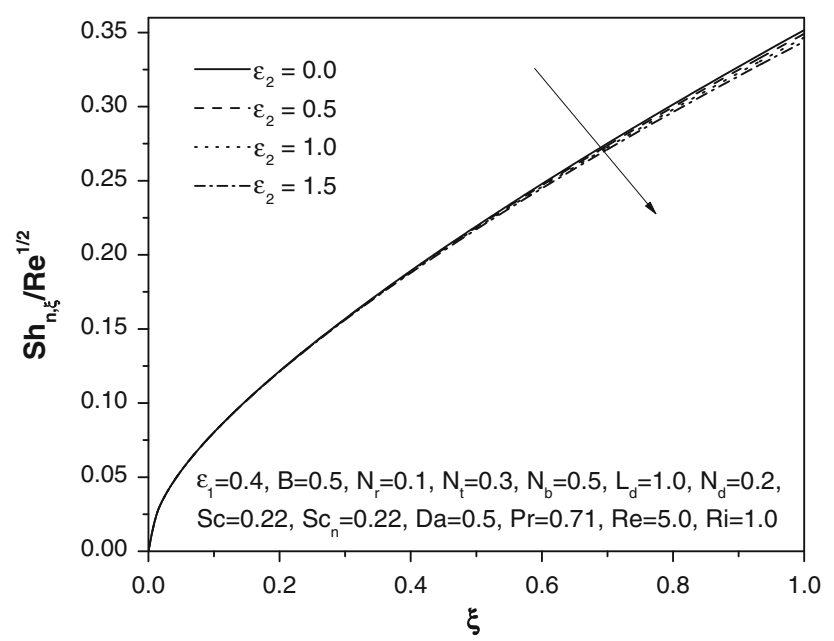

Fig. 14 Variation of local nanoparticle transfer coefficient with solutal stratification parameter

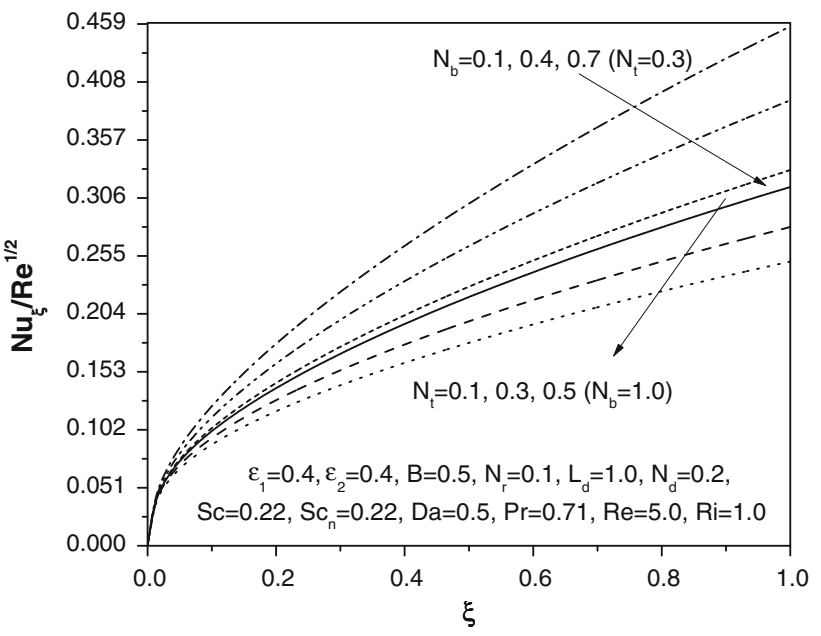

Fig. 15 Variation of local heat transfer coefficient with thermophoresis and Brownian motion parameters

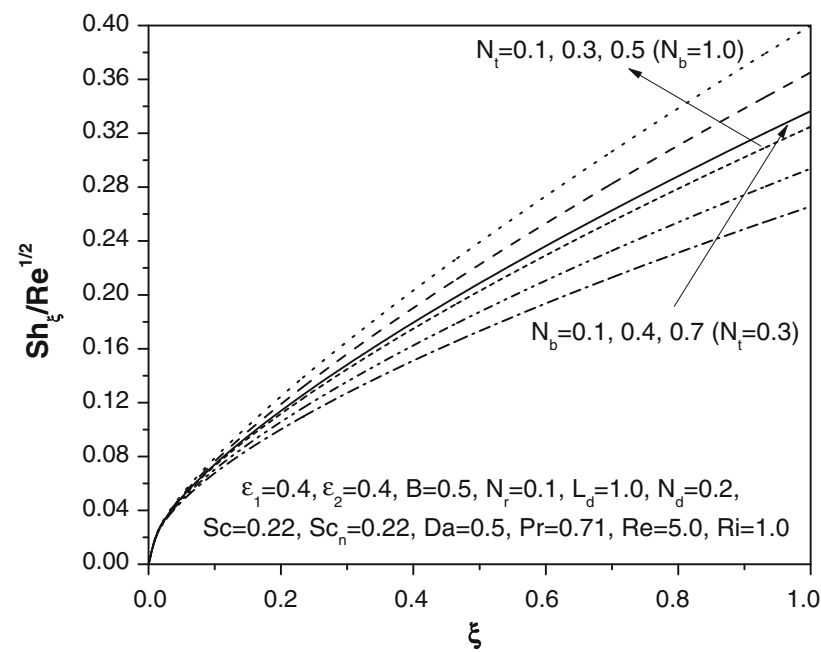

Fig. 16 Variation of local mass transfer coefficient with thermophoresis and Brownian motion parameters

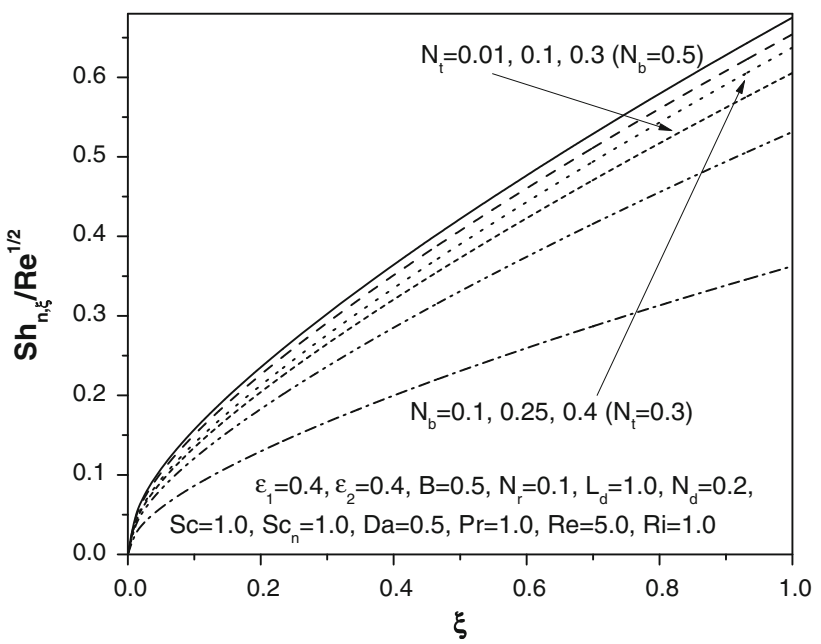

Fig. 17 Variation of local nanoparticle transfer coefficient with thermophoresis and Brownian motion parameters

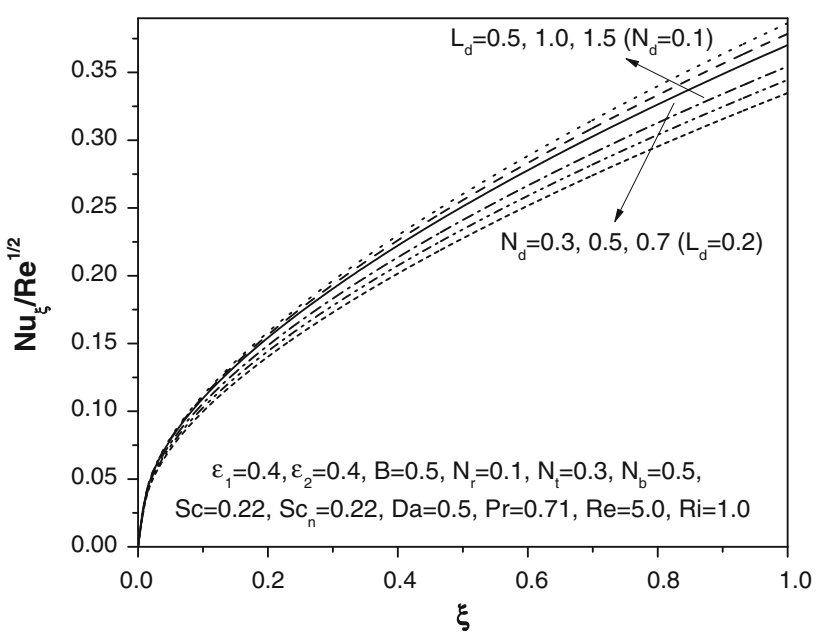

Fig. 18 Variation of local heat transfer coefficient with $L_{\mathrm{d}}$ and $N_{\mathrm{d}}$

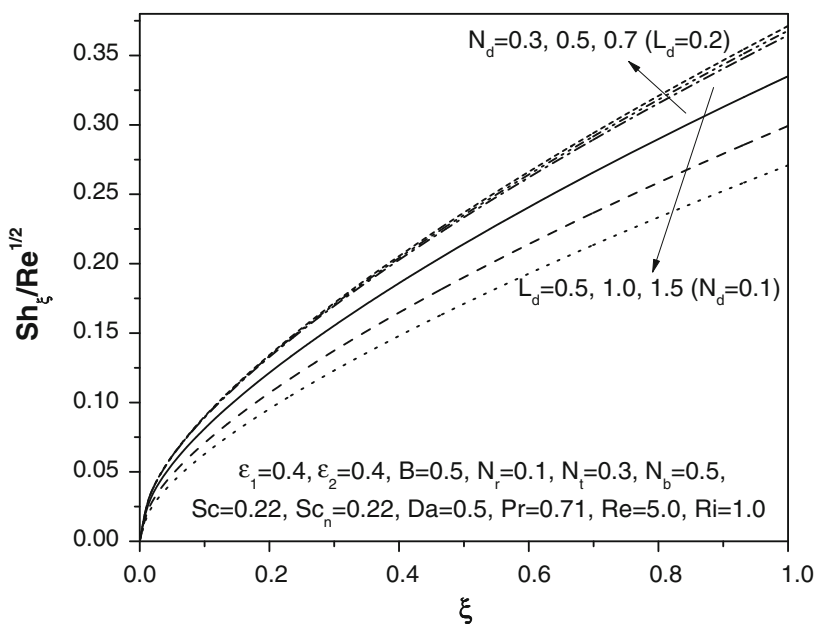

Fig. 19 Variation of local mass transfer coefficient with $L_{\mathrm{d}}$ and $N_{\mathrm{d}}$ 


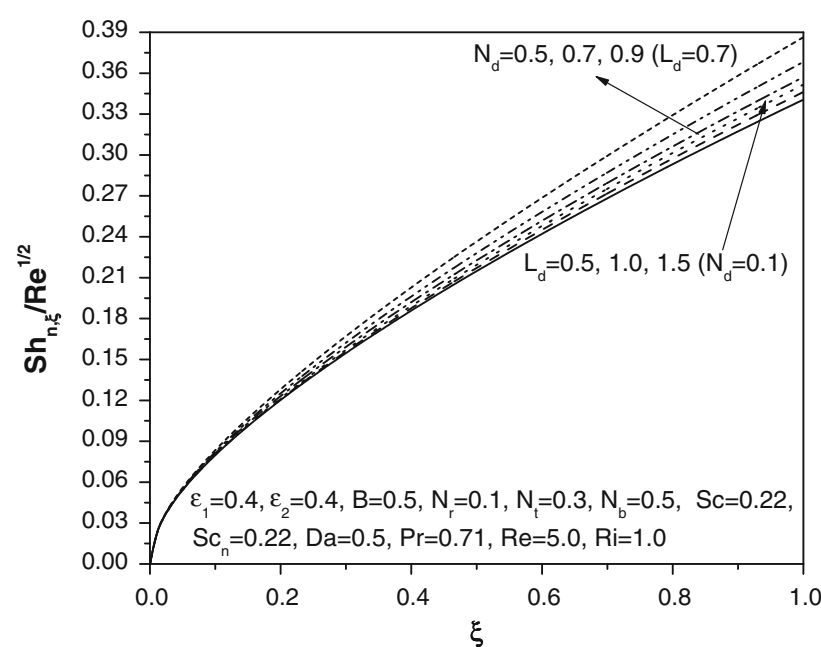

Fig. 20 Variation of local nanoparticle transfer coefficient with $L_{\mathrm{d}}$ and $N_{\mathrm{d}}$

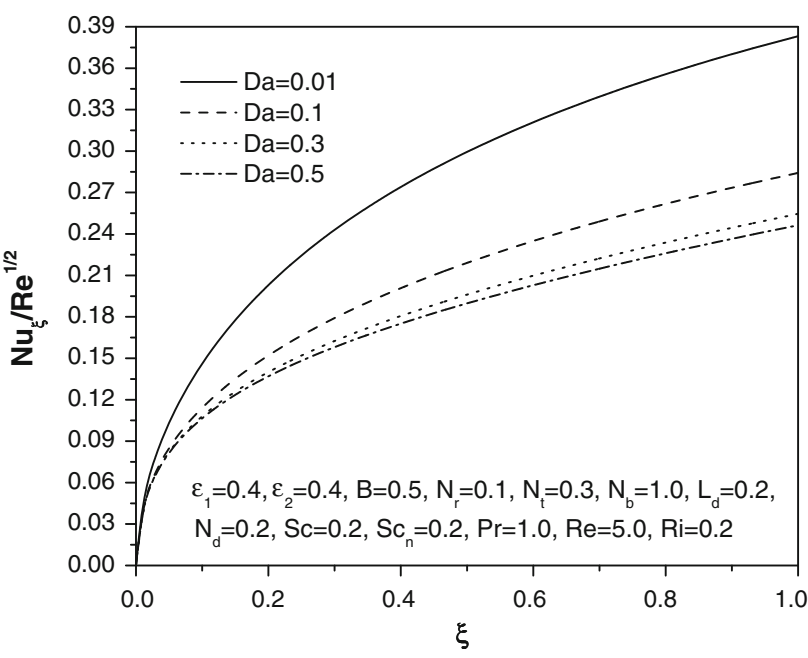

Fig. 21 Variation of local heat transfer coefficient with $D a$

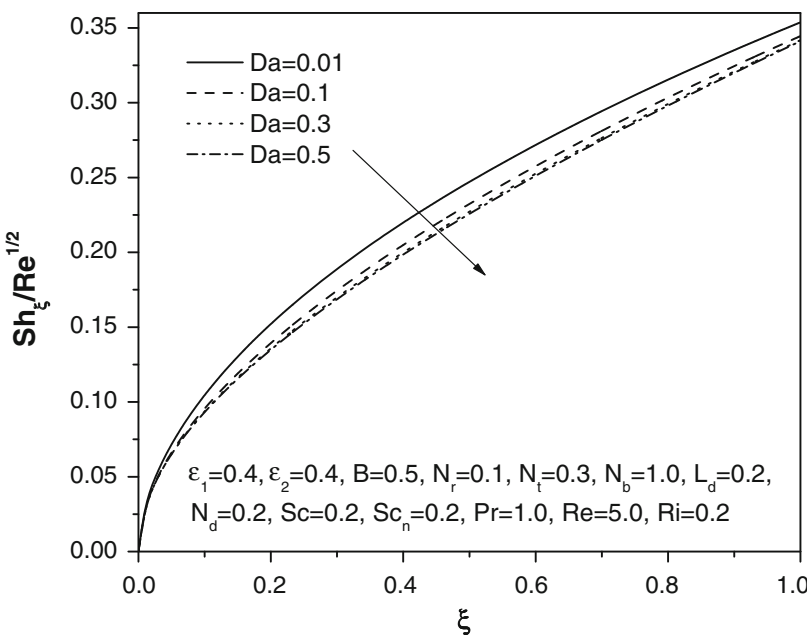

Fig. 22 Variation of local mass transfer coefficient with $D a$

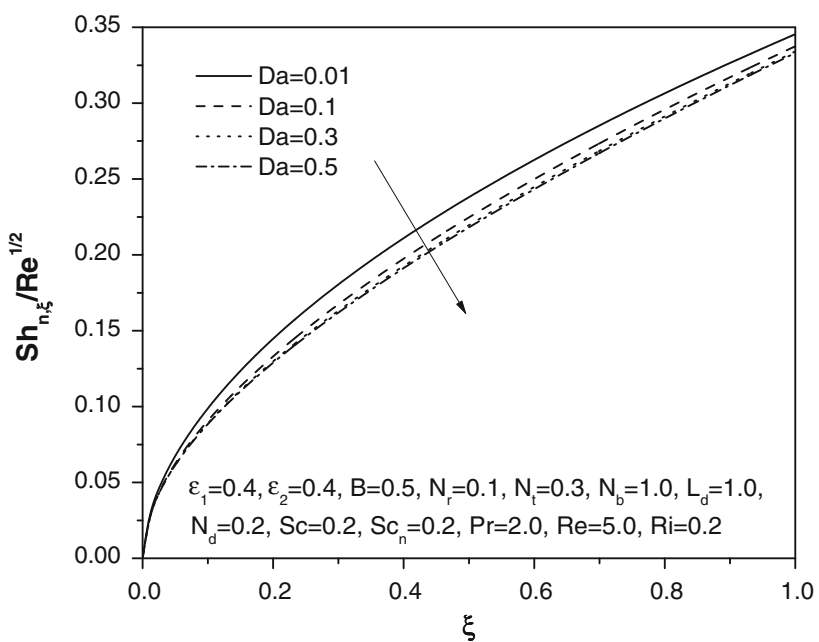

Fig. 23 Variation of local nanoparticle transfer coefficient with $D a$

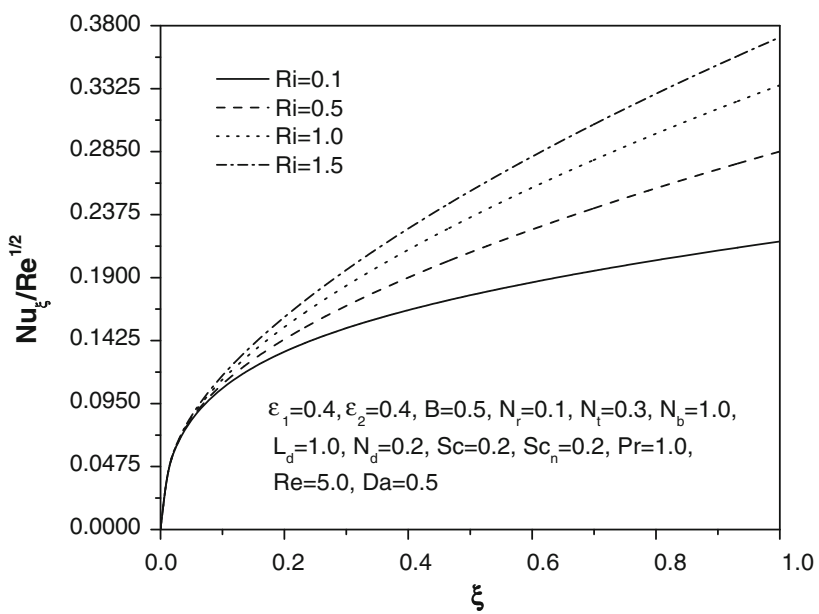

Fig. 24 Variation of local heat transfer coefficient with mixed convection parameter

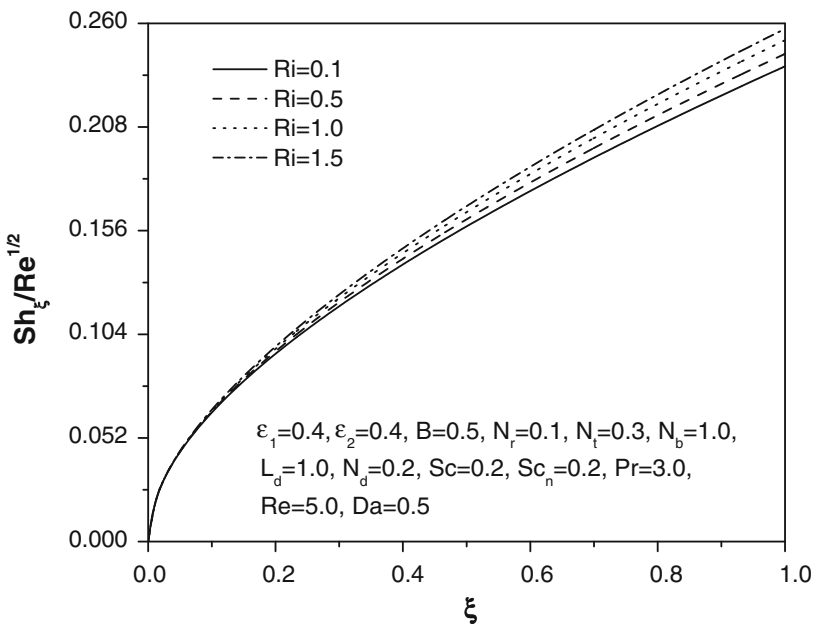

Fig. 25 Variation of local mass transfer coefficient with mixed convection parameter 


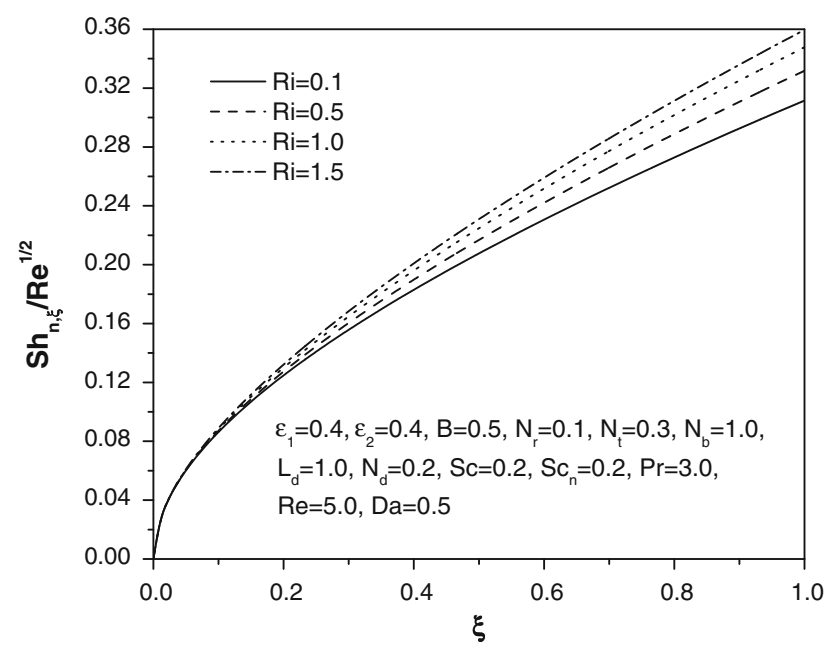

Fig. 26 Variation of local nanoparticle transfer coefficient with mixed convection parameter

nanofluids. The plate is subject to a uniform and constant wall heat, mass and nanoparticle fluxes. The governing equations are reduced to a system of non-similar parabolic equations for which numerical solutions have been presented for the wide range of parameters.

- An increase in the thermal stratification parameter $\varepsilon_{1}$, reduces the velocity, temperature and local heat, mass and nanoparticle transfer coefficients but enhances the concentration and nanoparticle fraction.

- The higher value of solutal stratification parameter $\varepsilon_{2}$ results in a lower velocity, concentration and local heat, mass and nanoparticle transfer coefficients but a higher temperature, nanoparticle fraction.

- The influence of Brownian motion parameter is to reduce the heat transfer rate and to enhance mass and nanoparticle transfer rates.

- The presence of thermophoresis effect is significantly decreased the heat transfer rate and increased mass and nanoparticle transfer rates.

- It is observed that the Dufour-solutal Lewis and modified Dufour numbers have significant influence on heat, mass and nanoparticle transfer rates.

- The local heat, mass and nanoparticle transfer rates are significanlty decreased with increase of Darcy number, but are enhanced with the increase of mixed convection parameter.
Open Access This article is distributed under the terms of the Creative Commons Attribution License which permits any use, distribution, and reproduction in any medium, provided the original author(s) and the source are credited.

\section{References}

Buongiorno J (2006) Convective transport in nanofluids. ASME J Heat Transfer 128:240-250

Cebeci T, Bradshaw P (1984) Physical and computational aspects of convective heat transfer. Springer, Verlag, New York

Chamkha AJ, Abbasbandy S, Rashad AM, Vajravelu K (2013) Radiation effects on mixed convection about a cone embedded in a porous medium filled with a nanofluid. Meccanica 48:275-285

Choi SUS, Zhang ZG, Yu W, Lockwood FE, Grulke EA (2011) Anomalously thermal conductivity enhancement in nanotube suspension. Appl Phys Lett 79:2252-2254

Das SK, Choi SUS, Yu W, Pradeep T (2007) Nanofluids: science and technology. Wiley. New Jersey

Gianluca P, Samuel P, Sen M (2011) Nanofluids and their properties. Appl Mech Rev 64:030803

Kakac S, Pramuanjaroenkij A (2009) Review of convective heat transfer enhancement with nanofluids. Int J Heat Mass Transfer 52:3187-3196

Lee SL, Chen TS, Armaly BF (1987) Mixed convection along vertical cylinders and needles with uniform surface heat flux. J Heat Transfer 109:711-716

Nazar R, Tham L, Pop I, Ingham DB, (2011) Mixed convection boundary layer flow from a horizontal circular cylinder embedded in a porous medium filled with a nanofluid. Transp Porous Med 86:517-536

Rahman MM, Eltayeb IA (2013) Radiative heat transfer in a hydromagnetic nanofluid past a non-linear stretching surface with convective boundary condition. Meccanica 48:601-615

RamReddy Ch, Murthy PVSN, Chamkha AJ, Rashad AM (2013) Soret effect on mixed convection flow in a nanofluid under convective boundary condition. Int $\mathrm{J}$ Heat Mass Transfer 64: 384-392

Rana P, Bhargava R, Beg OA (2012) Numerical solution for mixed convection boundary layer flow of a nanofluid along an inclined plate embedded in a porous medium. Computers Math Appl 64:2816-2832

Rohni AM, Ahmad S, Merkin JH, Pop I (2013) Mixed convection boundary-layer flow along a vertical cylinder embedded in a porous medium filled by a nanofluid. Transp Porous Med 96:237-253

Rosca AV, Rosca NC, Grosan T, Pop I (2012) Non-Darcy mixed convection from a horizontal plate embedded in a nanofluid saturated porous media. Int Commun Heat Mass Transfer 39:1080-1085

Rosmila AB, Kandasamy R, Muhaimin I (2012) Lie symmetry group transformation for MHD natural convection flow of nanofluid over linearly porous stretching sheet in presence of thermal stratification. Appl Math Mech Engl Ed 33:593-604 